\title{
Administered Item
}

National Cancer Institute

\section{Source}

National Cancer Institute. Administered Item. NCI Thesaurus. Code C42613.

A registry item for which administrative information is recorded in an Administration Record. 